\title{
Beitrag zur Anwendung des Tuberculins bei Lupus erythematodes und Lupus vulgaris.
}

Ton

\author{
Dr. Alfred Staub.
}

(Vortrag, gehalt. in Aerzte-Verein zu Posen am 7. April 1891.)

Meine Herren! Ich wollte mir erlauben, Ihre Aufmerksamkeit auf eive Erkrankung der Haut hinzulenken, welche man erst in den 40er Jahren dieses Jahrhunderts zu studiren angefangen, die aber seitdem das dermatologische Interesse wach erhalten und in diesen letzten Monaten, in welchen die Koch'sche Injectionsbehandlung in ihre Rechte getreten ist, uns lebhaft beschäftigt hat. Ich meine den Lupus erythematodes. Es ist dies eine chronische Lrkrankung der Haut, die mit bes mnderer Vorliebe im Gesicht auftritt und ihren Namen der Aehnlichkeit verdankt, welche die von ihr ergriffenen Hanttheile mit den von Lupus vulgaris befallenen aufweisen. Ich erlaube mir, Ihnen diese beiden Patienten vorzustellen. Der Eine hat, wie Sie sehen, diese beiden Fruptionen der linken Gesichtsseite; der eine Fleck von etwa Pfenniggrösse am linken Augenlide, von etwas hellrother Farbe, mässiger Infiltration, der Rand etwas erhaben, der Grund ein wenig vertieft, mit einem Schüppchen bedeckt; der zweite Fleck an der Nase, etwas grösser, intensiver roth, auch hier der Rand wallartig erhaben, sich über das Niveau des Plaques erhebend; auf dem flachen Grunde erkennen Sie theils fettige Schuppen, theils sehen Sie die offenen, klaffenden Mündungen der Talgdrüsen. Diese beiden Flecke bestehen seit Monaten in dieser Grösse und Ausdehnung; diese Flecke haben niemals genässt, waren niemals exulcerirt, sie 
verursachten dem Kranken nur ein geringes Picken, Stechen und Jucken; abgesehen von der Eintstellung keine Beschwerden. Ein dritter, ähnlicher, aber kleinerer Fleck ist von Herrn Sanitätsrath Samter, dessen Freundlichkeit ich die Zuweisung dieses Patienten verdanke, am oberen Augenlide derselben Gesichtsseite beobachtet worden, und in dessen Behandlung unter Anwendung von Blei-Acetat geschwunden, mit Hinterlassung einer leichten und unbedeutenden Röthe, während die anderen Flecken persistirten. Der Kranke, sonst gesund, zeigt auf der Gesichtshaut eine starke fettige Secretion der Talgdrüsen; auf der Haut des Rückens zahlreiche Comedonen und Acue-Pusteln. Der zweite Kranke bietet viel ausgedehntere Veränderungen auf seiner Gesichtshaut. Der Kranke sieht ein wenig entstellt aus; Sie sehen hier eine Reihe ron glatten, weissen, glänzenden Hautpartien abwechselnd mit einer Menge ron kleinen, linsen- bis pfenniggrossen, rothen, etwas erhabenen Plaques. Ich beobachte diesen Kranken, welchen ich der Liebenswürdigkeit des Herrn Oberstabsarzt Mäder verdanke, seit einer Reihe von Monaten; diese weissen, narbigen Stellen, die Sie jetzt sehen, sind aus ebensolchen entzündlichen Plaques hervorgegangen. Diese selbst ähneln in ihrer Configuration denen des anderen Kranken. Es handelt sich ủberall um kleine, rothe Scheiben, deren Rand etwas höher als das Centrum ist, welches selbst mit einem Schüppchen bedeckt ist. Aber wenn Sie diesen Kranken genauer betrachten, so fallen Ihnen noch mehrere Momente auf; erstens die lebhatte Röthe und Vascularisirung der Gesichtshaut, soweit sie von der Erkrankung noch nicht getroffen ist; zweitens an mauchen Stellen, z. B. auf der Nase, das lebhafte Hervortreten von Mitessern, Comedonen; drittens sehen Sie hier einen kleinen, frischen Fleck, der erst in den letzten Wochen zum Vorschein gekommen ist; er zeigt eine leichte Röthe und sieht etwas stachlig aus; wenn Sie genau hinsehen, bemerken Sie, besonders bei Tageslicht, auch bei diesem Kranken die weit klaffenden Mündungen der Talgdrüsen.

Wenn Sie eine Diagnose dieser Haut-Affection bei beiden Kranken stellen sollen, so werden Sie bei nur oberflächlicher Betrachtung geneigt sein, dieselbe für lupös zu halten; denn 
wie beim Lupus handelt es sich hier um chronisch entzündliche Infiltrate in der Haut, welche sehr lange bestehen und mit entstellender Narbenbildung abheilen, und das hat wohl Thompson-Parkes veranlasst, die Affection als Lupus superficialis zu beschreiben. Und doch gibt es eine Reihe von Momenten, durch welche sich die beiden Erkrankungen klinisch streng von einander unterscheiden lassen. Sie werden nirgends die kleinen Lupus-Knötchen, weder im Infiltrat, noch in der Umgebung desselben sehen, welche die charakteristischen Merkmale des Lupus vulgaris sind. Sie werden niemals sehen, dass diese Affection zur Ulceration, zur Geschwürsbildung führt, und Sie werden niemals sehen, wie entstellend die Krankheit auch im Gesicht wirken mag, dass sie in die liefe greift, die Knorpel usurirt, wie es der Lupus macht. Dagegen erkennen Sie an der begleitenden Seborrhoe, sowie an dern Hervortreten der Talgdrüsen in den Plaques das ätiologische Agens recht gut. Aetiologisch sind diese beiden Erkrankungen vollständig verschieden; denn der Lupus rulgaris ist, wie Sie wissen, eine tuberculöse Irkkrankung, welche durch Bildung miliarer Tuberkel in dem Gewebe der Cutis, durch das Wachsthum und die Metamorphose dieser Tuberkeln entsteht. Hingegen handelt es sich bei unserem Lupus erythematodes um eine Erkrankung, welche von den 'lalgdrüsen der Haut ansgeht. Es bilden sich entzündliche Infilitrate in der Umgebung der Hautdrüsen, welche zu einer langsamen Schrumpfung des Cutis-Gewebes führen. Diese Mitbetheiligung der Drüsen ist klinisch nicht in allen Stadien der Krankheit gleichmässig gut ausgesprochen; in den jüngeren Flecken besser als in den älteren, in denen es zu einer Verödung der Drüsen und Schrumpfung des Gewebes gekommen ist. In den ersten Fällen, welche Hebra, einer der ersten Erforscher dieses Krankheitsbildes, beschrieben hat, war die Betheiligung der Talgdrïsen so stark, dass er ihr den Namen Seborrhoea congestiva gab, indem er sie als eine fettige Hypersecretion der Hautdrüsen mit Congestion beschrieb. Schon ror ihm batte biett seine Fälle als Erythème centrifuge beschrieben, indem er auf die in centrifugaler Richtung sich ausdehneude Röthung, wie sie auch dieser Kranke bot, bei dem sie sich in Schmetterlingsflügelform von einer Backe über die 
Nase zur anderen Backe zog, besonderen Werth legte. Später hatte Cayenave, der den Uebergang dieser rothen Stellen in Narben beobachtete, eine Vervollständigung des klinischen Bildes gegeben, die wir seit ihm mit dem Namen Lupus erythematodes bezeichnen. Diese Affection, welche das Allgemeinbefiuden sonst ganz unbeeinträchtigt lässt, kann, wie Kaposi gezeigt hat, plötzlich durch universelle erysipelartige Ausbrüche gefährlich werden. - Aber wir wollen uns heute nicht mit den weiteren klinischen Eigenthümlichkeiten der Affection und auch nicht mit der Behandlung, die wir bisher anzuwenden pflegten, aufhalten. Sie wissen, seitdem in den letzten Monaten des vorigen Jabres die Koch'sche Lymphe zur Behandlung tuberculöser Affectionen zur Anwendung kam, man auch eine Reibe von anderen, nicht tuberculösen Erkrankungen als Versuchsobject benutzte. Man hat nun auch eine Reihe von Erkrankungen der Haut mit Koch'scher Lywphe behandelt, um zu sehen, ob das Mittel bei denselben irgend eine Reaction erzeugte. Und hier kam nun der Lupus erythematodes in erster Linie in Betracht. Erstens einmal gerade wegen der Aehnlichkeit, die er mit Lupus vulgaris hat; und zweitens, weil es in verschiedenen Ländern noch einzelne Forscher gibt, welche diese Erkrankungsform, wenn auch nicht für direct tuberculös, doch für eine "Abart" des tuberculösen Lupus halten. Von Hamburg, von Berlin, Breslau und Wien sind in diesen letzten Monaten ganz verschiedene Resultate berichtet worden. Arning beobachtete zwei Fälle; in beiden erhielt er auf Injection geringer Dosen eine starke allgemeine Reaction; in dem einen Fall war das Fieber mit allgemeinem Erythem der Haut des Körpers und des Gesichtes verbunden, so dass in der allgemeinen Röthe des Gesichtes die einzelnen Flecke weniger deutlich hervortraten; in dem anderen Falle war gar keine Reaction auf der Haut vorhanden. Lewin berichtet über einen Fall, in welchem er, ganz wie bei Lupus vulgaris, stark entzündliche Schwellung, Exsudation und Krustenbildung erhielt; doch wurde dieser Fall ron anderen Dermatologen, die ihn sahen, als nicht typischer Fall von L. erythematodes erklärt. Kaposi berichtete über zwei Fälle, von welchem der eine keine Reaction gab, der andere sehr starke Allgemein- und sehr starke örtliche Reac- 
tion mit Anschwellung und Verkrustung der Plaques zeigte und so schön und glatt abheilte, wie keiner der vielen LupusFälle, die er nach Koch behandelt hatte. Ich habe nun seit zwei Monaten ebenfalls bei Lupus eryth. Injectionen gemacht, mehr in der Absicht, die physiologische Wirkung des Mittels zu studiren, als in der Hoffnung, Heilerfolge zu erzielen. Ich habe zunächst bei diesem Kranken mit den ausgedehnten Erkrankungserscheinungen folgende Beobachtungen gemacht: Die erste Dosis von $1 \mathrm{Mgr}$. hatte bei dem sonst gesunden und kräftigen Mianne gar keine Wirkung; die zweite Einspritzung ron $5 \mathrm{Mgr}$., mehrere Tage später applicirt, ebenfalls nicht; der Patient empfand ein paar stunden nach der Injection einen leichten Frost. der schuell verschwand, ohne Temperaturerhöhung. Acht Tage später wurde ihm 1 Ctgr. am Vormittag injicirt. Es erfolgte auch darauf keine Temperaturerhöhung, wohl aber stellte sich am Abend eine ganz excessiv starke Hyperhidrosis ein. Stundenlang perlte der Schweiss in grossen und kleinen Tropfen im Gesicht und an Körper. Das Gesicht zeigte sich. stark geröthet. Die Röthe trat auch auf den glatten, weissen, narbigen Stellen auf; die Plaques waren succulenter und traten schärfer hervor. Diese Reaction war bei Weitem nicht zu vergleichen mit der erysipelartigen, starken Schwellung, welche der Lupus vulgaris nach den Koch'schen Injectionen zeigt. Es zeigte sich auch keine Spur von Nässen und Krusteubildung, sondern nur eine stärkere Prominenz der erkrankten Theile. Schon am nächsten Tage hatte die Gesichtshaut wieder ihre frühere Färbung und die Plaques bildeten sich ein wenig zurück, so dass sie ein wenig kleiner waren, als vor der Einspritzung. Dies veranlasste mich, da das Allgemeinbefinden des Patienten am Tage der Einspritzung nur wenig litt und bald nach der Einspritzung ganz normal war, acht Tage später eine Injection von $2 \mathrm{Cgr}$. zu machen. Und dieses Mal erhielt ich die interessanteste Reaction. Die 'Temperatur stieg auf $38 \cdot 2$, die Gesichtshant war wieder etwas geröthet, etwas geschwellt, die erkrankten Partien traten deutlicher und stärker hervor; es zeigte sich wieder ein starker Schweiss, besonders am Gesicht, aber auch am Körper; an der Haut des Halses und des Rumpfes zeigte sich eine ganz merkwürdige Strichelung: sie schien wie beklext 
mit rothen Linien, die am Nacken radienförmig verliefen, ebenso an der Brust, an beiden Mammae, hinten am Rücken mehr in Längsrichtung, an der Grenze von Brust und Bauch mehr quer verliefen. Die Röthe verschwand beim Druck, es handelte sich also um eine Hyperämie von Bl utgefässen der Haut. Sie wissen, dass verschiedene, theils morbillöse, masernähnliche, theils scharlachähnliche und andere erythematöse Exantheme als Folgeerscheinung der Tuberkulin-Injectionen beschrieben worden sind, während in den bisherigen Publicationen über ein solches Exanthem noch nicht berichtet wurde. Auch nach dieser Injection bildeten sich die Lupus-Plaques ein wenig zurück, so dass ich die Hnffnung hegte, durch Fortsetzung der Injectionen ein Schwinden der Flecke zu erzielen, während bisher durch Anwendung ron Causticis wohl eine Heilung der Plaques erzielt worden war, ohne dass ich, wie in anderen glücklicheren Fällen, ein Aufschiessen neuer Plaques verhindern konnte. Die günstige bisherige Einwirkung des Mittels bei diesem Kranken veranlasste mich, auch bei dem zweiten Kranken die Injectionsbehandlung einzuleiten. In der That schien nach der ersten Injection von 3 Mgr. eine leichte Rückbildung der Plaques einzutreten, ohne dass am Injectionstage mehr als eine leichte Röthe im Gesicht als Reaction sich zeigte. Eine zweite Injection von $7 \mathrm{Mgr}$. erzeugte ebenfalls keine allgemeine oder locale Reaction, die Affection selbst steht auf derselben Stufe wie nach der ersten Injection. Inzwischen habe ich bei dem ersten Kranken meine Versuche fortgesetzt. Es wiederholte sich dasselbe Spiel; ohne Temperaturerhöhung zeigte sich wieder ein Erythem des Gesichtes, Anschwellen der Plaques, welche nach der Injection kleiner schienen, wie zuvor. Eine ähnliche Reaction beschrieben Schweninger und Bazzi. Obwohl wir nun eine ganz interessante Reaction, die Hyperhydrosis und eine leichte Schwellung am Tage der Injection mit einem darauf folgenden Abschwellen und Abblassen zu verzeichnen gehabt haben, kann ich über eine vollständige Ausheilung trotz $\mathrm{zweimonatlicher}$ Behandlung nicht berichten. - Als Gegenstïck gegen diese Reaction bei Lupus erythematodes möchte ich Ihnen noch diese Patientin mit Lupus vulgaris vorführen, welche eine sehr interessante Reaction zeigte, die einige anregende Ideen für die Frage 
der ganzen Koch'schen Behandlung bietet. Diese Patientin, welche seit länger als einem Jahrzehnt an einer sehr unangenehmen Form ron Lupus, einem exulcerirenden Lupus der Nase, der Nasenhöhlen und beider Gesichtshälften litt, ist durch Behandlung mit Causticis vor Jahresfrist von mir gebeilt worden. In Anbetracht des grossen Umfanges, welchen die lupöse Erkrankung hatte, war die Patientin mit dem Erfolge recht zufrieden. Es bildete sich eine schöne, glatte Narbe, nur in der Mitte beider Backen etwas strahlig eingezogen. Der äusserste Theil der Nasenspitze war bereits durch den Lupus zerstört. Seit Jahresfrist zeigte sich bei der Patientin auch kein Recidiv. Jetzt in diesem Monat zeigten sich an drei Stellen kleine, verdächtige Nachschübe, welche sich aber nur durch eine geringe Abschuppung der Oberhaut über ihnen verriethen. Der ganze übrige Theil war schön, glatt und weiss, zeigte nirgends ein suspectes Infiltrat; er war also noch in demselben guten Heilungszustande wie vor einem Jahre. Wir bezeichnen einen Lupus als geheilt, wenn weder in der nach der Zerstörung des Lupus entstandenen Narbe, noch in ihrer Umgebung irgend eine Spur eines verdächtigen Infiltrates zu sehen ist, und wenn wir beobachten können, dass auch im Laufe von mehreren Monaten und Jahren ein solches Infiltrat nicht eintrat. Doch wir wissen es sehr wohl, dass auch in dieser anscheinend schönen und fehlerfreien Narbe kleine Tuberkelreste übrig sein können, die nach längerer Zeit wieder zur kräftigen Lntwicklung kommen. Von dieser Art der Heilung bitte ich sehr bestimmt eine zweite Form ron Heilung zu unterscheiden, welche man jetzt nach den Koch'schen Injectionen häufig beobachten kann, bei welcher die lupösen Geschwüre zuheilen, aber in der sich bildenden Narbe noch reichliche und deutliche tuberculöse Infiltrate zu erkennen sind. Unser Fall gehörte nun mehr zu der ersten Art ron geheiltem Lupus, obwohl ich nicht zweifelte, dass in dieser grossen Narbe, auch abgesehen von den drei kleinen suspecten Stellchen, kleine, dem Auge noch unsichtbare, tuberculöse Infiltrate zurückgeblieben wären, welche vielleicht in Monaten oder Jahren mehr oder weniger umfangraiche locale Recidive verursachen würden.

Ich injicirte nun dieser sonst kräftig und gat gebauten 
Patientin eine Dosis von $2 \mathrm{Mgr}$. Es trat eine stürmische Reaction ein; es entwickelte sich eine sehr lebhafte, erysipelastige Anschwellung in dem ganzen Umfang der Narbe. Die Narbe wurde sebr roth, gespannt, unter lebhaftem Brennen; dis Nasenhöhle schwoll besonders in der linken Hälfte stark an; und dieser Zustand hielt unter lebhafter Betheiligung des Allgemeinbefindens, starker Schwäche, Mattigkeit mit Ohnmachten und Appetitlosigkeit zwei Tage an. Die drei verdächtigen Stellen bedeckten sich nach dem Abschwellen der afficirten Partien mit kleinen Krusten, und jetzt, 13 Tage nach der Einspritzung, ist die übrige Narbe noch etwas geröthet, hat ihre frühere weisse, glatte Beschaffenheit noch nicht erreicht. Dagegen zeigt sie eine Anzahl verdächtiger Infiltrate in der Tiefe; die Krusten auf den suspecten Partien sind noch vorhanden.

M. H.! Als die ersten Versuche mit Tuberkulin-Injectionen gemacht wurden, erregte es gerade besonderes Aufsehen, dass schon geheilte Lupus-Partien nach der Injection stark anschwollen und zu erneuter Krustenbildung und dann wieder zur Vernarbung gelangten. Die Lymphe schien die verborgensten Tuberkelherde aufzusuchen und zur Elimination zu bringen. Nun, wir wissen nach den bisherigen Erfahrungen, dass es wohl gelingt, einen exulcerirenden Lupus zur oberflächlichen Vernarbung zu bringen, dass wir aber die Infiltrate in der Tiefe damit nicht beseitigen können. Wenn wir in solchen Fällen die Injection fortsetzen, so bringen wir wobl auch diese jetzt zum Yorschein gekommenen Infiltrate, welche durch die Injection neu mobil gemacht worden sind, noch zur Verkrustung und dann wieder zu einer oberflächlichen Vernarbung; aber einen definitiven Erfolg erzielen wir damit nicht. Nach einer zweiten Injection von $2 \mathrm{Mgr}$, , die wieder äusserst stürmische Reactionen hervorrief, heilten bei unserer Patientin die Krusten, aber die lupösen Infiltrate in der Narbe traten noch deutlicher hervor. Ich möchte mir daher erlauben, die These aufzustellen, dass erstens das Koch'sche Mittel im Stande ist, bei den ulcerirenden Formen des Lupus eine oberflächliche Vernarbung zu erzeugen, und dies können wir mit den bisherigen Mitteln, ohne so starke Alteration des Allgemeinbefindens, ebenfalls erreichen. Aber doch wird da, wo ein Lupus exul- 
cerans ausgedehnte Körperstellen betrifft, diese Methode schneller zum Ziele führen, als die bisher iiblichen localen Lupusbehandlungen, insbesondere beim Lupus der Schleimhäute. Aber ich möchte nicht rathen, wie es von manchen Seiten vorgeschlagen wurde, solche Lupus-Fälle, welche durch andere Methoden zur Heilung gebrach worden sind, mit Injectionen zu behandeln; nachträglich denn hier ist das Koch'sche Mittel im Stande, in der kürzesten Zeit und auf die acuteste Weise den Process anzufachen, ohne doch die Fähigkeit zu haben, den entstaudenen Brand vollständig und definitiv zu löschen. Es ist gar kein Zweifel, dass das Koch'sche Mittel eine ganz specifische physiologische Wirkung auf den tuberculösen Process ausuibt; aber durch diese interessante physiologische Wirkung ist es noch nicht befahigt, auch wirkliche Heilresultate zu erzielen. Das Mittel hat aber $z$ weiten fuir den Lupus eine eminente diagnostische Wirkung. Es gibt bäufig zweifelhafte Fälle, bei denen auch ein geäbter Blick die Infferential-Diagnose zwischen lupösen und anderen Processen nicht zu stellen im Stande ist. Und in solchen Fällen verbleibt der Koch'schen Lymphe ein dauernder Werth in der Dermatologie.

Ich hatte mich der Hoffnung hingegeben, welche jetrt auch in Berlin auf dem Chirurgen-Congress zum Ausdruck gekommen ist, dass es vielleicht gelingen müsste, durch Anwendung einer anderen Methode als der bisher gebräuchlichen, welche in der Anwendung fortschreitend stärkerer Dosen bestand, zum Ziele zu gelangen. Ich meinte, durch Anwendung minimaler Dosen, welche in langen Zwischenrüumen auf einander folgen, eine günstigere Beeinflussung des Lupus erzielen zu können. Indess bin ich auch von dieser Hoffnung zurückgekommen, sowoll nach meinen eigenen lirfahrungen, als nach den Veröffentlichungen aus dem Hôpital St. Louis in Paris, an welchem die Ductoren Besnier, Hallopeau, Vidal u. A. mit ziemlich kleinen Dosen (schwächer, als die bei uns angewandten) in nicht zu kurzen Zwischenräumen während mebrerer Wochen auch nicht zu besseren Resultaten gekommen sind.

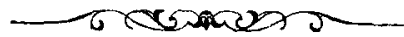

